\title{
Narrativa
}

narrativa

nuova serie

Nuova serie

39 | 2017

Nuove frontiere del Sud

\section{Una nuova allegoria ipermoderna: il parco turistico a tema storico}

Daniela Carmosino

\section{(2) OpenEdition}

1 Journals

Edizione digitale

URL: https://journals.openedition.org/narrativa/702

DOI: $10.4000 /$ narrativa. 702

ISSN: 2804-1224

Editore

Presses universitaires de Paris Nanterre

\section{Edizione cartacea}

Data di pubblicazione: 1 décembre 2017

Paginazione: 139-148

ISBN: 978-2-84016-289-6

ISSN: $1166-3243$

Notizia bibliografica digitale

Daniela Carmosino, «Una nuova allegoria ipermoderna: il parco turistico a tema storico», Narrativa

[Online], 39 | 2017, online dal 01 décembre 2021, consultato il 14 janvier 2022. URL: http://

journals.openedition.org/narrativa/702 ; DOI: https://doi.org/10.4000/narrativa.702

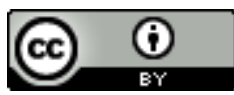

Narrativa est mise à disposition selon les termes de la Licence Creative Commons Attribution 4.0 International. 


\section{Una nuova allegoria ipermoderna: il parco turistico a tema storico}

$\circlearrowleft$ e è vero che la vexata quaestio dell'identità del Sud d'Italia anima da secoli, ormai, il dibattito tra studiosi di vari settori, è anche vero che, nell'ultimo decennio, all'interno di questo stesso dibattito, si sono imposti due specifici temi.

Il primo è il necessario riconoscimento dell'identità plurale del Sud, ovvero delle innegabili difformità che, a dispetto di certe letture "geneticamente" unificanti, esistono all'interno della convenzionale nozione di Sud, per diversa ascendenza storico-culturale o per diverso sviluppo economico.

Il secondo e altrettanto necessario riconoscimento è quello della collocazione di questi territori all'interno dello scenario del mondo globalizzato, così che diventi impossibile ragionare di identità al di fuori di tale contesto.

Oggi non è più possibile prescindere dalla nuova cartografia su base economico-culturale tracciata negli ultimi decenni dalla Globalizzazione, dalla New Economy, dal Tecnocapitalismo, dal Pensiero Unico. Se la nuova mappatura trasforma il mondo in Mercato, ove quest'ultimo viene a coincidere con l'intero perimetro della superficie "mondanizzata", un criterio di elaborazione identitaria può esser rappresentato dalla minore o maggiore capacità d'un territorio di aderire al modello economico-culturale elaborato e proposto/imposto dal Centro. Ovvero, dai paesi capitalistici dell'area nord-occidentale.

Categoria non più geografica, quella del "periferico" finisce così per imporsi con nuovi tratti e per individuarsi in virtù del rapporto (prevalentemente di antagonismo o di resilienza) che intrattiene con la Globalizzazione.

La trasformazione del rapporto Centro/Periferia ha avuto inizio con la trasformazione della società moderna in società della comunicazione. L'ingresso nel postmoderno si presenta, infatti, anche come deflagrazione del Centro e della sua moderna, "eurocentrata", griglia interpretativa in una miriade di punti di vista, di fonti di informazione e di elaborazione di stili di vita, valori, obiettivi. 
In questo senso, il postmoderno inaugura una vera e propria moltiplicazione dei centri, tutti egualmente legittimi all'interno della nuova, eterarchica cartografia del mondo rappresentata dalla Rete.

Prezioso può, allora, risultare lo spostarsi da una lettura privilegiante l'aspetto dello sviluppo economico-industriale e tecnologico verso una lettura in chiave culturale. Imprescindibile, quest'ultima, dal momento che il Mercato si regge necessariamente sulla propedeutica creazione di una classe di nuovi consumatori informata alla cultura del consumo. In tal modo, ciò che dal punto di vista del Mercato si colloca in posizione decentrata o marginale, dal punto di vista culturale esibirà invece una maggior vivacità e prontezza nell'interpretare originalmente (magari in versione glocal) il modello proposto, o nello sviluppare pensiero critico a partire da questo.

Un esempio per tutti: i nuovi luoghi e non-luoghi della socializzazione e i nuovi modelli relazionali che ne conseguono, innestandosi in aree dalla forte, persino ingombrante, identità storico-culturale, danno vita a originali configurazioni ossimoriche, efficacissime nell'evidenziare i limiti sia dell'arroccamento su posizioni di difesa della tradizione (local), sia sull'accoglimento passivo e acritico dei nuovi stili di vita (global).

Laddove, insomma, la marginalità rispetto al processo economico si traduca in una più produttiva posizione a latere, ecco che si genera un pensiero critico nato dall'osservare, sperimentare quotidianamente, quali buman consequences, gli effetti positivi come pure i limiti, i rischi, le derive di un processo storico di cui pure quel territorio è partecipe e con cui interagisce e reagisce. E questa mi pare, oggi, una possibile interpretazione della cultura del Sud d'Italia entro la nuova cartografia del mondo.

Infatti, ciò che in questa prospettiva viene a delinearsi è un Sud che accoglie e sperimenta nuove e stimolanti forme di socializzazione e di relazione con l'Altro (crossfertilization), nuovi codici comunicativi e nuovi linguaggi e poi nuove forme di impiego, precariato e/o di sfruttamento del lavoro legate alla decolonizzazione, all'emigrazione di massa o alla delocalizzazione.

Nel percepirsi, dunque, all'interno d'una storia comune e d'una comune cartografia culturale e, al tempo stesso, nel mantenere la giusta distanza critica in virtù d'una collocazione decentrata rispetto al "motore" del Mercato; nel continuo incontro con l'Altro da Sé che proprio il procedere della Storia continuamente impone, incontro che non sia consumo ma esperienza trasformativa: in questo mi pare offra al Sud d'Italia la possibilità di costruire e affermare la propria identità, attraverso una precisa individuazione, che tuttavia la mantenga all'interno d'una storia condivisa. 
In questa prospettiva, i nuovi narratori del Sud ${ }^{1}$ raccontano la realtà "aggiornata al presente" della loro terra: narratori ipermoderni, inclini a accogliere sulla pagina i temi, i simboli, le icone del presente facendoli reagire con i contesti meridionali, se è vero che i modi di rappresentazione prediletti oscillano dal realismo venato d'ironia al grottesco in funzione demistificante, una delle tematiche predilette è, invece, proprio la Storia.

Una Storia ingombrante, certo, una storia imposta, monumentale, che pervade l'immaginario collettivo del Sud: ma anche una storia del territorio in grado di arginare o ridimensionare la postmoderna percezione d'una temporalità tutta appiattita sul presente, tutta sviluppata in superficie e priva di profondità.

Da Giuseppe Montesano a Giorgio Vasta, da Franco Arminio a Carmen Pellegrino, da Antonio Pascale a Massimo Maugeri a Livio Romano, quasi tutti i nuovi narratori del Sud si cimentano, così, nel racconto dell'ossimorica convivenza o dello stridere spaesante fra le tracce d'una Storia percepita come rimosso e la superficie smaltata d'un presente assoluto, da consumare in velocità.

Ecco allora che il parco a tema storico si ribalta di segno, ergendosi quale mostruosa allegoria del passaggio dall'esperienza del passato al suo consumo turistico.

Per parco a tema storico si intendano quei mega-complessi edilizi pressoché autosufficienti, destinati al soggiorno turistico e al divertimento, che presentano una specifica caratteristica: riproducono gli scenari di uno o più epoche storiche, sia con un sommario allestimento di setting storico-tematici, sia con la messa in scena, tramite attori e comparse, dei principali eventi storici delle epoche citate. Lontano dal puro divertissement del citazionismo postmoderno, lontano dal gioco svincolato da ogni finalità, il parco a tema storico non ha, tuttavia, neppure un fine didattico. Come nei più cupi scenari di Adorno, questo tipo di progetti informano le sole logiche del Mercato: il fruttuoso investimento, ha infatti lo scopo di sedurre e dilettare masse di turisti-consumatori pronti ad immergersi acriticamente in un viaggio virtuale all'interno dei più corrivi stereotipi storicoartistici. Ora, in una società in cui tutti siamo consumatori e ogni cosa è prodotto, Storia e Cultura subiranno anch'esse un processo di trasformazione in prodotti di largo, facile, im-mediato e rapido consumo, e come tali verranno spettacolarizzati, immessi sul mercato, "messi a reddito".

1. S'intende qui quella categoria di scrittori di cui si è descritto il programmatico insorgere contro gli stereotipi del Sud in CArmosino, Daniela, Uccidiamo la luna a Marechiaro, Roma, Donzelli, 2009. 
Eleggere a protagonista d'un romanzo il parco a tema storico, così come fanno il catanese Massimo Maugeri e il salentino Livio Romano, significherà, allora, tematizzare l'attuale mutata percezione e del passato storico e di quello biografico. Come dire, un ulteriore sviluppo rispetto alla crisi del logos storico e dell'idea di storia come corso unitario, già ampiamente descritta nel corso del Novecento. L'attuale percezione "arcipelagica" dello spazio, infatti, vanifica anche la possibilità di produrre, generare Storia, dunque di costruire la propria identità, individuale e collettiva, dall'interno di questa. Non solo. Sulla scorta di Gilles Lipovetzsky e Jean Serroy: "l'individuo ipermoderno non si accontenta dei piaceri: chiede anche di essere protagonista [...] di partecipare".

Infine: se oggi, nell'epoca dell'attention economy, il consumatore è assuefatto a un generalizzato processo di spettacolarizzazione che lo iperstimola, di certo più sensorialmente che intellettivamente, ciò a cui sempre più si sta disabituando, invece, è il processo di simbolizzazione della realtà.

Un'interessante proposta di lettura del fenomeno ci proviene dalla psicoanalisi di Massimo Recalcati, altro teorico della svolta ipermoderna. Nel suo L'uomo senza inconscio ${ }^{3}$ Recalcati osserva come oggi, nell'epoca della progressiva dissoluzione di ogni paterna auctoritas, d'ogni limite, d'ogni confine e della spaesante libertà di informazione, consumo, azione, il soggetto non ha più desideri da trasformare in "rimosso", poi destinati all'inevitabile ritorno attraverso il linguaggio "altro", simbolico, dell'Inconscio: il nuovo imperativo categorico, infatti, è quello di godere ${ }^{4}$, godere consumando il presente. In ossequio a ciò, oggi il soggetto non cerca, nell'incontro col passato, quell'esperienza dell'Altro da sé produttrice di identità, ma si ferma a un edonistico, narcisistico, onanistico piacere tratto dal consumo agile, veloce, superficiale.

Come dire che l'individuo presenta oggi un problema di "analfabetismo": disabituato ai processi di simbolizzazione e interpretazione della realtà, non è più in grado di riconoscerli, non sa distinguere la finzione dalla realtà, non sa cogliere né apprezzare la messa in forma estetica e la simbolizzazione proprie dei processi artistici né (complici, andrà detto, certi destoricizzanti orientamenti nella formazione delle nuove generazioni) sa interpretare l'opera, come pure,

2. Lipovetzsky, Gilles, Serroy, Jean, La Cultura-Mondo. Risposta a una società disorientata, Milano, O barra O, p. 115.

3. Recalcati, Massimo, L'uomo senza inconscio. Figure della nuova clinica psicoanalitica, Milano, Raffaello Cortina editore, 2010.

4. Un godere che ha ormai poco a che vedere con la lacaniana lettura di Cartesio e dell'imperativo categorico di Kant. 
fenomeno assai più pericoloso, la realtà in genere, alla luce della sua genesi e nel suo contesto di riferimento.

Della difficoltà di interpretare e narrare tanto la storia collettiva quanto la propria biografia, come pure dell'evaporare dei tratti distintivi, ovvero dei confini, tra esperienza reale, esperienza virtuale e finzionalità, ragionano Maugeri e Romano nei loro romanzi, e lo fanno attraverso quell'allegoria della nuova percezione della storia che è il parco turistico a tema storico.

Il parco ideato da Maugeri, il Trinacria Park che dà il nome al romanzo, coincide con l'intero perimetro di un immaginario isolotto difronte alla costa siciliana, Montelava, nello stesso modo in cui il Mercato Globale coincide col mondo.

Il mega-complesso edilizio esibisce subito le stimmate dell'autosufficienza (logistica e metaforica) rispetto al contesto

super attrezzato, innovativo, dotato di un'area servizi, due ampi parcheggi, magazzini, uffici amministrativi, centri benessere, sei sale cinematografiche, due teatri, una discoteca da cinquemila posti, ristoranti, teatri, studi televisivi, un centro medico, cinque grandi alberghi con seimila posti letto, sale congresso, negozi, piscine, campi da calcio, da tennis, da golf, uno stadio da diecimila posti, parchi acquatici, un aeroporto e una darsena $a^{5}$.

Ma ciò che a noi più interessa è quell'area storica chiamata Little Sicily, una sorta di sintesi o semplificazione della Sicilia, che riproduce solo le piazze e i monumenti di maggior rilievo, "o quantomeno", interviene la voce narrante, virando al sarcastico, "di quelli più meritevoli da un punto di vista turistico". "In tal modo", prosegue la voce narrante,

il visitatore può farsi un'idea generale delle meraviglie storico architettoniche della Sicilia $[\ldots]$ Può anche fotografarle e riprenderle. Può leggere o sentire $[\ldots]$ notizie sulle storie e sulle leggende [...] Il visitatore può beneficiare di una visione d'insieme, per poi procedere all'incontro dal vivo con i luoghi che ritiene più interessanti ${ }^{\top}$.

L'esperienza d'una realtà finzionale "dal vivo" diventa così sinonimo di esperienza del vero: e difatti, nel parco, tutto ciò è "credibile, realistico, perfetto nella sua veridicità"».

5. Maugeri, Massimo, Trinacria Park, Roma, E/O, 2013, p. 116.

6. Ibid., p. 12.

7. Ibid., p. 117.

8. Ibid., p. 118. 
Ciò che qui mi pare assai ben rappresentato è l'esito di un passaggio importante: il prevalere del criterio tematico a discapito della contestualizzazione storica caratterizzante l'organizzazione del reale. È evidente infatti come, attraverso uno strategico citazionismo destoricizzante, documentalità, virtualità, finzionalità vengano dispiegate tutte sulla medesima superficie da un soggetto-consumatore che continua a mantenere pieno possesso di quelle facoltà, proprie della prospettiva albertiana e umanistica, di "com-prendere" il mondo in una visione d'insieme orientata, però, da e verso un preciso focus che seleziona il materiale visivo e lo inquadra entro una griglia interpretativa: quella, appunto, informata alla logica del Mercato.

Ecco allora che, all'interno del parco, avremo ampie "zone a tema che rappresentano dal vivo grandi eventi della storia siciliana": dallo sbarco dei Mille a Il Gattopardo, dallo sbarco americano nella seconda guerra al mito greco delle Gorgoni. Nel romanzo di Maugeri, d'altronde, proprio come nella percezione e nella produzione mediatica del reale, fictum e factum, collocabili in posizione ontologicamente paritaria, sconfinano incessantemente l'uno nell'altro, come pure si confondono e confondono il lettore in un barocco e allucinato gioco di specchi. In quest'ottica andranno letti anche commenti e dichiarazioni, di chiarissima ascendenza pirandelliana, quali "è tutto così falso. Ma è la vita in fondo a essere falsa" o "È impossibile rappresentare davvero la realtà"

E non è un caso se, nel gran calderone in cui mito, leggenda e documento condividono il medesimo statuto di "credibilità", la storia della Sicilia non venga narrata, bensì messa in scena attraverso una rappresentazione turistica in cui muoversi spensieratamente, una virtualità immune dal calore potente e dall'energia dell'esperienza emotiva reale, tuttavia resa sufficientemente emozionante tramite la spettacolarizzazione. In questo senso l'Etna riprodotto in scala, alto trenta metri e che erutta una lava sintetica, si offre davvero quale allegoria nell'allegoria.

Non ci stupisca, allora, che, in una realtà tutta dispiegata in orizzontale, la Storia in accezione moderna possa aver voce solo come rimosso, solo attraverso rappresentazioni simboliche, di secondo grado, giochi di specchi, allusioni, metafore. È unicamente attraverso la speculare immagine di Montelava che possiamo guardare e raccontare la "pietrificante" realtà siciliana: ne è una chiara spia tutta l'aggettivazione utilizzata per descrivere l'immaginario isolotto, che "ha subito l'assalto di vacanzieri senza scrupoli $[. .$.$] ha patito un'edilizia$

9. Ibid., p. 42.

10. Ibid. 
selvaggia $[\ldots]$ si è piegat $[\mathrm{o}]$ al volere tornacontista dei tanti politici responsabili, di imprenditori privi di scrupoli e poesia"11. E ancora: "Montelava è sempre stata considerata come un'isola abbandonata a se stessa, vittima dell'incuria, dell'avidità, del guadagno di pochi a discapito dell'interesse collettivo. Un'isola indicata come esempio negativo, zavorra, pattumiera" ${ }^{12}$.

Ciò non vuol dire che Maugeri estrometta il senso della Storia dal suo romanzo: solo lo fa emergere come rimosso, appunto, come disturbo, come sintomo utile quanto invalidante: "è un capogiro", "è amore per il luogo in cui si è nati", "nostalgia per un passato irrisolto, senso di colpa per scelte incerte e opinabili", "malattia che scorre nel sangue di chi percepisce la ricchezza della storia, la bellezza della natura, il peso delle tradizioni, la zavorra delle contraddizioni. È un virus $[\ldots]$ che non aiuta a vivere bene, ad essere moderni ed efficienti" ${ }^{\prime \prime}$.

Dalla postmoderna difficoltà o resistenza a cogliere la dimensione della profondità, tout court, dunque quella della Storia, consegue la difficoltà a organizzare la narrazione della propria biografia e la propria identità: il vissuto personale fatica a trovare una sistemazione logica, almeno quanto fatica a farsi spazio nelle pagine di Maugeri, ove compare di rado e in corsivo, talvolta in dialetto, par excellence linguaggio dell'autenticità: "Che razza di uomo sono io? Per quanto tempo dovrò essere contenitore di queste viscere di carta?... Mi sento inadeguato, qualunque cosa faccia"14. E ribadisce in un ulteriore passaggio: "Che ci faccio qui? [...] È questo che desidero veramente?"15.

D'ascendenza tutta pirandelliana, infine, sarà la più tradizionale, espressionistica, deformazione dei tratti somatici, sintomo d'una chiara distonia:

Mentre parla sa di mostrare un'aria accigliata. Lo sa, perché in giornate come questa, quando il ventre genera l'inferno, difficilmente riesce a dissimulare il furore della disapprovazione. Ecco il furore gli si appiccica alla faccia gli si sparge sui lineamenti, gli condiziona l'espressione ${ }^{16}$.

Temi e questioni simili li ritroviamo anche nell'allegorica rappresentazione di Livio Romano, Per troppa luce ${ }^{17}$.

11. Ibid., p. 41.

12. Ibid., p. 127.

13. Ibid., p. 17.

14. Ibid., p. 62.

15. Ibid., p. 63.

16. Ibid., p. 43.

17. Romano, Livio, Per troppa luce, Milano, Sironi, 2016. 
Qui il parco turistico a tema storico si presenta solo in forma di progetto ideato da tre affaristi cialtroni e da un sedicente architetto. Ciò che invece Romano ci offre sono, da un lato, la realtà, il modello societario e relazionale, gli stili di vita e di consumo, la logica di mercato, insomma, da cui progetto trae vita e nutrimento; dall'altro, la logica antagonista del comitato di cittadini che vuole proteggere ${ }^{18}$ l'area del Salento destinata al parco e già coltivata a ulivi secolari.

Le pagine di Romano riescono a far scorrere rapidissimamente sulla rètina una carrellata di simboli, icone, prodotti cult del Mercato, o ancora sulla scia di Lipovetzsky e Serroy, della Cultura-Mondo ${ }^{19}$ : dai più tipici prodotti di consumo, cui il soggetto demanda il compito di attribuirgli identità, sino a certe modalità di consumo adottate indifferentemente per cibi, abiti, location, persone, sesso. Il primo potente risultato è così quello di farci esperire, più che raccontarci, una versione grottescamente amplificata dell'infelice, forzato, bulimico, indifferenziato consumo della realtà che oggi sta sostituendo l'esperienza.

Interessante è poi, anche qui, la tematizzazione della difficoltà a percepire $\mathrm{e}$ ordinare il passato cogliendolo nella sua estensione spazio-temporale, a livello di storia del territorio quanto di biografia personale.

Negli ultimi vent'anni, circa, la terra di Puglia, la cui sopravvivenza è dipesa in gran parte dalla coltivazione di ulivi secolari, qui chiaro simbolo della storia e della tradizione, è stata scoperta dal turismo di massa. L'opposizione tra le due fonti di sostentamento, agricolo e turistico, viene ipostatizzata attraverso il dialogo tra uno degli affaristi implicati nel progetto e il proprietario delle terre su cui dovrebbe ergersi Neripoli, il parco turistico a tema storico che, per dirla con uno degli speculatori, lascerà "alla città il più grande monumento italiano. Altro che chiese, statue, piazze e palazzi”"20.

Il parco si chiamerà Neripoli, come l'antica città su cui dovrebbe essere edificato, e sarà

una vera città monumento, una cosa fore ti capu, tutto ricostruito alla perfezione, arrivi e ti trasporti in un'altra epoca, anzi un'altra sera, dell'uomo primitivo, li Messapi, là, come si chiamano loro, vivevano qua no? Dice l'architetto di sì. Ha costruito città egiziane, città romane, città mesopotamiche, città arabe, dappertutto in America. Tutto intorno, alberghi, discoteche e casinò ${ }^{21}$.

18. Cfr. ID., Porto di mare, Milano, Sironi, 1991, romanzo anch'esso centrato sul tema dell'edilizia turistica megalomane e devastante le coste del Sud d'Italia.

19. Cfr. Lipovetzsky, Gilles, Serroy, Jean, La Cultura-Mondo. Risposta a una società disorientata, cit.

20. Romano, Livio, Per troppa luce, cit., p. 28.

21. Ibid., pp. 28-29. 
Anche Romano, come Maugeri, rimescola le carte tra vero e virtuale, confondendo sempre i due piani, laddove l'esperienza d'una realtà finzionale "dal vivo"' diventa di nuovo sinonimo di esperienza del vero: "mica ste cose virtuali di oggigiorno. Noi il viaggio nel passato, alla gente, lo faremo fare sul serio, dal vivo"22.

La Storia e l'identità del territorio trovano, così, spazio solo in forma turistica, "gadgettizzata", commercializzabile, "per comunicare all'intero pianeta che il Salento è la nuova meta esclusiva" 23 .

Oppure come logo o marchio: se il proprietario terriero difende i suoi ulivi come "vecchi secoli e secoli. Monumenti a loro volta" ${ }^{24}$, l'affarista mira a valorizzare ulivi e tombe come "marchio della nostra civiltà Messapica", "addobbo", come "spruzzatina di storia con la S maiuscola"25.

E anche qui la percezione superficiale, alla lettera, del passato storico, trova il suo corrispettivo nella sfera personale, in una confusa, epidermica e nevralgica percezione del vissuto biografico.

Anche in Romano, il soggetto che abita il tempo presente non riesce a produrre una narrazione del proprio passato dotata di senso e progettualità. Protagonista di tale disagio la coppia sentimentale formata da Antonio, ispettore del lavoro e Simona, avvocato civilista, entrambi invischiati nelle disavventure di Neripoli: non percependo con chiarezza le ragioni dei propri comportamenti e l'origine dei propri desideri, non decodificando il proprio inconscio, questi non sono in grado di vivere il presente in piena consapevolezza e alla luce della propria storia, della propria identità costruita su base biografica.

E non è un caso che anche Romano, per la rappresentazione dei movimenti fisici e dei moti interiori di Antonio e Simona farà ricorso a due elementi teatrali posti "fuori scena", ovvero "osceni": la parentetica del "a parte" e il corsivo della didascalia.

Ti amo ti amo ti amo, Antonio (si alra, va ad abbracciarlo, lui resta bloccato come un baccalà ...)

Simo, ora lasciamo stare proprio, sai? Ti prego, mettiti in moto. Da questo momento io e te non ci parliamo più. (Non si capacita delle parole che gli spifferano dai denti, vorrebbe dire l'esatto contrario, Vorrebbe invitarla da quel momento in poi a restare insieme per tutta la vita) ${ }^{26}$.

22. Ibid., p. 31 .

23. Ibid., p. 29.

24. Ibid.

25. Ibid., p. 30.

26. Ibid., pp. 218-219. 
E ancora:

Devo attraversare il deserto, Simona. Devo farlo da solo. Presente quel senso che non trovi più??

Non importa Antonio [...] È che tu non ci sei, non puoi esserci. Tu dividi anche te stesso. E sarebbe invece ora che ti raccogliessi in una persona sola ${ }^{27}$.

In conclusione, è vero che il logos della moderna storiografia ha preso a dissolversi già con l'avvento del postmoderno. Oggi, però, molte sono le derive e le trasformazioni di un processo di destoricizzazione dell'immaginario collettivo. Tanto che, nell'epoca attuale, che fa seguito al postmoderno e che alcuni designano col termine di ipermodernità, si assiste a una sorta di ritorno del rimosso, almeno in campo artistico, individuabile in certo "ritorno alla realtà" e all'impegno etico.

Un impegno che si traduce in una rappresentazione del presente che non sia mera referenzialità o piatta mimesi, bensì nasca da un pensiero critico su quello stesso presente: un presente assoluto, postmoderno, che ci propone quale valore primario l'incessante e spasmodico consumo del nuovo.

Ed è entro questo scenario che la nuova narrativa del Sud si impegna a rappresentare, con grande lucidità, la propria terra, facendola assurgere a sede di sperimentazione di un comune presente storico, prefigurandone, non di rado, $\mathrm{i}$ futuri scenari.

Risultato cui, d'altronde, dovrebbe aspirare la migliore letteratura.

Daniela CARMOSINO Università del Molise 